\title{
SCHUR'S DOUBLE CENTRALIZER THEOREM FOR TRIANGULAR HOPF ALGEBRAS
}

\author{
MIRIAM COHEN, DAVIDA FISCHMAN, AND SARA WESTREICH
}

(Communicated by Lance W. Small)

\begin{abstract}
Let $(H, R)$ be a triangular Hopf algebra and let $V$ be a finitedimensional representation of $H$. Following Manin we imitate the standard algebraic constructions in order to define the relativized notions of $R$-universal enveloping algebras of $R$-Lie algebras and the $R$-Lie algebra $\mathrm{gl}_{R}(V)$. Using Majid's "bosonization" theorem and the above we prove an $R$-analogue of Schur's double centralizer theorem.
\end{abstract}

\section{INTRODUCTION}

Let $V$ be a finite-dimensional vector space over a field $k$ of characteristic 0 . Let $m$ be a positive integer. Then the symmetric group $S_{m}$ acts on $V^{\otimes m}$ (via the twist map), and the Hopf algebra $U(\mathrm{gl}(V))$ acts on $V^{\otimes m}$ via its comultiplication. In this setting (an equivalent form of) Schur's double centralizer theorem states that $S_{m}$ and $U(\mathrm{gl}(V))$ are mutual centralizers in $\operatorname{End}_{k} V^{\otimes m}$. This result was generalized to super vector spaces by [BR], and both these situations were translated into a Hopf algebraic setting and thus given a natural proof by the second author [Fi].

In this paper we deal with a triangular Hopf algebra $\left(H_{1}, R\right)$ over a field $k$ [D] and the category $H_{1}$ Mod of left $H_{1}$-modules.

In $\S 2$ we construct for any $R$-Lie algebra [Man] the $R$-analogue of a universal enveloping algebra $U_{R}(L)$ and show in Theorem 2.6 that $U_{R}(L)$ is a Hopf algebra in the category ${ }_{H_{1}}$ Mod (it is not an ordinary Hopf algebra though).

In $\S 3$ we consider a finite-dimensional $V$ in the category ${ }_{H_{1}}$ Mod. First we define $\mathrm{gl}_{R}(V)$ the $R$-analogue of $\mathrm{gl}(V)$, an $R$-Lie algebra. Utilizing Majid's recent "bosonization" theorem [Maj2] we form $H=U\left(\mathrm{gl}_{R}(V)\right) \# H_{1}$, an ordinary Hopf algebra. Next we consider actions of $H$ and $k S_{m}$ on $V^{\otimes m}$.

On one hand $k S_{m}$ acts on $V^{\otimes m}$ via the braiding inherited from $R$; on the other hand $H$ acts on $V^{\otimes m}$ via the comultiplication of $H$. In Theorem 3.8 we

Received by the editors March 5, 1992 and, in revised form, November 24, 1992.

1991 Mathematics Subject Classification. Primary 16W30.

The first and third authors' research was supported by the Basic Research Foundation administered by the Israel Academy of Sciences and Humanities. 
show that in characteristic 0 these representations of $k S_{m}$ and $H$ are mutual centralizers in End $_{k} V^{\otimes m}$, thus giving an $R$-analogue of Schur's theorem.

\section{Preliminaries}

Let $H$ be a Hopf algebra over a field $k$ with comultiplication $\Delta: H \rightarrow$ $H \otimes H$, counit $\varepsilon: H \rightarrow k$, and antipode $S: H \rightarrow H$. We use Sweedler's [S] "sigma notation" for $\Delta$; that is, $\Delta(h)=\sum h_{(1)} \otimes h_{(2)}$, for all $h \in H$.

Let $M={ }_{H}$ Mod denote the category of left $H$-modules. For each $V \in M$, End $_{k} V \in M$, where for each $f \in \operatorname{End}_{k} V$ and $h \in H$

$$
(h \cdot f)(v) \equiv \sum h_{(1)} \cdot\left(f\left(S\left(h_{(2)}\right) \cdot v\right)\right)
$$

Moreover, if $V, W \in M$, then $V \otimes W \in M$, where for each $v \otimes w \in V \otimes W$ and $h \in H$

$$
h \cdot(v \otimes w)=\sum h_{(1)} \cdot v \otimes h_{(2)} \cdot w
$$

The tensor algebra of $V, T(V)$, is thus an $H$-module algebra. From the properties of the comultiplication, ${ }_{H}$ Mod is a monoidal category. There does not usually exist, though, a categorical ( $H$-module) isomorphism between $V \otimes W$ and $W \otimes V$ for $V, W \in{ }_{H} \operatorname{Mod}$. The usual twist map $\tau$ is not necessarily an $H$-module map. When the Hopf algebra is (quasi-) triangular such an isomorphism exists and ${ }_{H}$ Mod becomes a (quasi-) tensor category [Maj1].

Definition 1.3 [D]. A quasi-triangular Hopf algebra is a pair $(H, R)$, where $H$ is a Hopf algebra and $R=\sum R^{(1)} \otimes R^{(2)} \in H \otimes H$ satisfies the following (with $r=R)$ :

(1) $R$ is invertible,

(2) $\left(\Delta \otimes \mathrm{id}(R)=\sum R^{(1)} \otimes r^{(1)} \otimes R^{(2)} r^{(2)}\right.$,

(3) $\quad(\mathrm{id} \otimes \Delta)(R)=\sum R^{(1)} r^{(1)} \otimes r^{(2)} \otimes R^{(2)}$,

(4) $(\tau \circ \Delta)(h)=R \Delta(h) R^{-1}$ for all $h \in H$.

It is a consequence of the above that

$$
R^{-1}=\sum S\left(R^{(1)}\right) \otimes R^{(2)} \quad \text { and } \quad \sum \varepsilon\left(R^{(1)}\right) R^{(2)}=1 .
$$

$(H, R)$ is called triangular if $R^{-1}=\sum R^{(2)} \otimes R^{(1)}$. When $(H, R)$ is quasitriangular

$$
\Psi_{V, W}: V \otimes W \rightarrow W \otimes V \quad \text { given by } \quad v \otimes w \rightarrow \sum R^{(2)} \cdot w \otimes R^{(1)} \cdot v
$$

is an $H$-module isomorphism. Moreover when $(H, R)$ is triangular, it satisfies $\psi_{W, V} \circ \psi_{V, W}=\mathrm{id}$. Sometimes we write $\psi_{12}$ for $\psi_{V_{1}, V_{2}}$.

Note that when $H$ is a cocommutative Hopf algebra, $(H, 1 \otimes 1)$ is triangular and $\psi_{V, W}=\tau$. The rule of thumb in what follows is that whenever one needs to move a $v$ past a $w$ the switching is done via $\psi_{V, W}$. Typically we have

Definition 1.4 [CW, Maj2]. Let $A$ and $B$ be $H$-module algebras. Then $A \otimes B$ is an $H$-module algebra by defining the product

$$
\mu: A \otimes B \otimes A \otimes B \rightarrow A \otimes B, \quad a \otimes b \otimes c \otimes d \rightarrow \sum a\left(R^{(2)} \cdot c\right) \otimes\left(R^{(1)} \cdot b\right) c .
$$

Definition 1.5. $B \in{ }_{H}$ Mod is a Hopf algebra in the category if all its structure maps $\Delta_{B}, \mu_{B}, S_{B}, \varepsilon_{B}$, and $u_{B}$ (satisfying the usual Hopf algebra diagrams) are $H$-module maps. 
In particular, note that $H$ itself is usually not a Hopf algebra in the category ${ }_{H}$ Mod, although it is an ordinary Hopf algebra.

Majid [Maj2] has recently developed a process by which a Hopf algebra in the category ${ }_{H}$ Mod is twisted ("bosonized" in his terminology) to become an ordinary Hopf algebra. The "bosonization" theorem was motivated by Fischman's paper [Fi] which deals with supervector spaces (i.e., spaces constructed by "Bosons" and "Fermions"). Our present paper is a sequel to [Fi], heavily using the bosonization theorem.

We state this theorem in a particular setting (we do not state it in its full generality as it involves various concepts that are out of the scope of this paper).

Theorem 1.6 [Maj2, 4.1]. Let $\left(H_{1}, R\right)$ be a triangular Hopf algebra over $k$, and let $B$ be a Hopf algebra in the category ${ }_{H_{1}}$ Mod. If $\psi_{B, B} \circ \Delta_{B}=\Delta_{B}$ (i.e., $B$ is "cocommutative" in the category), then the algebra $H=B \# H_{1}$ is an ordinary triangular Hopf algebra, where (upon identifying $B$ with $B \# 1$ and $H_{1}$ with $1 \# H_{1}$ ) for all $b \in B$ and $h \in H_{1}$

$$
\begin{aligned}
& \hat{\Delta}(b \# h)=\sum\left(b_{(1)} \# R^{(2)} h_{(1)}\right) \otimes\left(R^{(1)} \cdot b_{(2)}^{\# h_{(2)}}\right), \quad \text { where } \Delta_{B}(b)=\sum b_{(1)} \otimes b_{(2)} \text {; } \\
& S(b \# h)=\sum S\left(h_{(2)}\right) u R^{(1)} \cdot S(b) \# S\left(R^{(2)} h_{(1)}\right), \quad \text { where } u=\sum S\left(R^{(2)}\right) R^{(1)} \text {; } \\
& \varepsilon(b \# h)=\varepsilon(b) \varepsilon(h), \quad u(1)=1 \# 1, \quad R_{B \# H_{1}}=R_{H_{1}} \text {. }
\end{aligned}
$$

The algebra structure on $B \# H_{1}$ is given as usual by

$$
(b \# h)\left(b^{\prime} \# h^{\prime}\right)=\sum b\left(h_{(1)} \cdot b^{\prime}\right) \# h_{(2)} h^{\prime} .
$$

\section{The $R$-UNIVERSAL ENVELOPING ALGEBRA}

Let $\left(H_{1}, R\right)$ be a triangular Hopf algebra. In this section we introduce the notion of an $R$-universal enveloping algebra of an $R$-Lie algebra. This enveloping algebra, unlike the usual one, is not an ordinary Hopf algebra but will turn out to be a Hopf algebra in the category $H_{1}$ Mod .

Following Manin [Man] we first define an $R$-Lie algebra to be an object $L$ in $H_{1}$ Mod together with an $H_{1}$-morphism [ , ] $R: L \otimes L \rightarrow L$ satisfying

$$
R \text {-anticommutativity: }\left[l_{1}, l_{2}\right]_{R}=-\sum\left[R^{(2)} \cdot l_{2}, R^{(1)} \cdot l_{1}\right]_{R} \text {, }
$$

$R$-Jacobi identity:

Let $\left\{l_{1} \otimes l_{2} \otimes l_{3}\right\}_{R}$ denote $\left[l_{1},\left[l_{2}, l_{3}\right]_{R}\right]_{R}, S_{312}=\psi_{12} \circ \psi_{23}$, and $S_{231}=\psi_{23} \circ \psi_{12}$ (where $\psi_{23}\left(l_{1} \otimes l_{2} \otimes l_{3}\right)=\sum\left(l_{1} \otimes\left(R^{(2)} \cdot l_{3}\right) \otimes\left(R^{(1)} \cdot l_{2}\right)\right)$,etc.). Then the following identity holds

$0=\left\{l_{1} \otimes l_{2} \otimes l_{3}\right\}_{R}+\left\{S_{312}\left(l_{1} \otimes l_{2} \otimes l_{3}\right)\right\}_{R}+\left\{S_{231}\left(l_{1} \otimes l_{2} \otimes l_{3}\right)\right\}, \quad$ for all $l_{i} \in L$.

Note that $[,]_{R}$ being an $H_{1}$-module homomorphism means via (1.2) that $h \cdot\left[l_{1}, l_{2}\right]_{R}=\sum\left[\left(h_{(1)} \cdot l_{1}\right),\left(h_{(2)} \cdot l_{2}\right)\right]_{R}$, for all $h \in H$.

Now one can form the derived $R$-Lie algebra from any $H_{1}$-module algebra $A$ be defining an "inner" $R$-Lie product [Man]

$$
[,]_{R}: A \otimes A \rightarrow A \quad \text { by }[a, b]_{R}=a b-\sum\left(R^{(2)} \cdot b\right)\left(R^{(1)} \cdot a\right) .
$$

This is a morphism in $H_{1}$ Mod (while the usual commutator is not, unless $R=$ $1 \otimes 1)$. Denote the derived $R$-Lie algebra by $A^{-}$. 
Let $\left(L_{i},[,]_{R}^{L_{i}}\right), i=1,2$, be $R$-Lie algebras. An $R$-Lie homomorphism is an $H_{1}$-module homomorphism $f: L_{1} \rightarrow L_{2}$ satisfying

$$
f\left(\left[l_{1}, l_{2}\right]_{R}^{L_{1}}\right)=\left[f\left(l_{1}\right), f\left(l_{2}\right)\right]_{R}^{L_{2}}, \quad \text { for all } l_{1}, l_{2} \in L_{1} .
$$

The following is an $R$ analogue of the classical notion.

Definition 2.3. Let $\left(L,[,]_{R}\right)$ be an $R$-Lie algebra. An $R$-universal enveloping algebra of $L$ is a pair $(U, u)$, where $U$ is an associative $H_{1}$-module algebra with $1, u: L \rightarrow U^{-}$is an $R$-Lie homomorphism, and the following holds: for any associative $H_{1}$-module algebra $A$ and any $R$-Lie homomorphism $f: L \rightarrow A^{-}$there exists a unique algebra homomorphism, which is an $H_{1}$-module homomorphism, $g: U \rightarrow A$, such that the following diagram commutes:

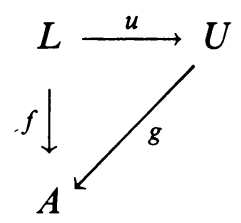

Proposition 2.4 (existence). Let $\left(L,[,]_{R}\right)$ be an R-Lie algebra; let $U(L)=$ $T(L) / I$, where $I$ is the ideal of $T(L)$ generated by

$$
\left\{l_{1} \otimes l_{2}-\sum R^{(2)} \cdot l_{2} \otimes R^{(1)} \cdot l_{1}-\left[l_{1}, l_{2}\right]_{R}, \text { for all } l_{1}, l_{2} \in L\right\} ;
$$

and let $u: L \rightarrow T(L) / I$ be the canonical map, $l \mapsto l+I=\bar{l}$. Then $(U(L), u)$ is an $R$-universal enveloping algebra for $L$.

Proof. First, $U(L)$ is an $H_{1}$-module algebra since $T(L)$ is by (1.2) and $I$ is $H_{1}$-stable, for

$$
\begin{aligned}
h \cdot\left(l_{1} \otimes\right. & \left.l_{2}-\sum R^{(2)} \cdot l_{2} \otimes R^{(1)} \cdot l_{1}-\left[l_{1}, l_{2}\right]_{R}\right) \\
= & \sum\left(h_{(1)} \cdot l_{1} \otimes h_{(2)} \cdot l_{2}-h_{(1)} R^{(2)} \cdot l_{2} \otimes h_{(2)} R^{(1)} \cdot l_{1}\right. \\
& \left.-\left[\left(h_{(1)} \cdot l_{1}\right),\left(h_{(2)} \cdot l_{2}\right)\right]_{R}\right) \\
= & \sum\left(h_{(1)} \cdot l_{1} \otimes h_{(2)} \cdot l_{2}-R^{(2)} h_{(2)} \cdot l_{2} \otimes R^{(1)} h_{(1)} \cdot l_{1}\right. \\
& \left.-\left[\left(h_{(1)} \cdot l_{1}\right),\left(h_{(2)} \cdot l_{2}\right)\right]_{R}\right) \in I .
\end{aligned}
$$

The rest is obvious.

Let us write $[$,$] for [,]_{R}$.

Proposition 2.5. Let $L_{1}$ and $L_{2}$ be $R$-Lie algebras. Then there exists an $H_{1^{-}}$ module algebra homomorphism

$$
g: U\left(L_{1} \oplus L_{2}\right) \rightarrow U\left(L_{1}\right) \otimes U\left(L_{2}\right) .
$$

Proof. Define $f: L_{1} \oplus L_{2} \rightarrow U\left(L_{1}\right) \otimes U\left(L_{2}\right)$ by $\left(l, l^{\prime}\right) \rightarrow \bar{l} \otimes 1+1 \otimes \bar{l}^{\prime}$. We claim that $f$ is an $R$-Lie homomorphism from $L_{1} \oplus L_{2}$ to $\left(U\left(L_{1}\right) \otimes U\left(L_{2}\right)\right)^{-}$. Recall that multiplication in $U\left(L_{1}\right) \otimes U\left(L_{2}\right)$ is

$$
(a \otimes b)\left(a^{\prime} \otimes b^{\prime}\right)=\sum a\left(R^{(2)} \cdot a^{\prime}\right) \otimes\left(R^{(1)} \cdot b\right) b^{\prime},
$$


for all $a, a^{\prime} \in U\left(L_{1}\right)$ and $b, b^{\prime} \in U\left(L_{2}\right)$. Well,

$$
\begin{aligned}
f\left[\left(l, l^{\prime}\right),\left(s, s^{\prime}\right)\right]^{L_{1} \oplus L_{2}} & =f\left([l, s]^{L_{1}},\left[l^{\prime}, s^{\prime}\right]^{L_{2}}\right) \\
& =\overline{[l, s]} \otimes 1+1 \otimes\left[\overline{\left.l^{\prime}, s^{\prime}\right]}=[\bar{l}, \bar{s}] \otimes 1+1 \otimes\left[\bar{l}^{\prime}, \bar{s}^{\prime}\right] .\right.
\end{aligned}
$$

Now,

$$
\begin{aligned}
& {\left[f\left(l, l^{\prime}\right), f\left(s, s^{\prime}\right)\right]^{\left(U\left(L_{1}\right) \otimes U\left(L_{2}\right)\right)^{-}} } \\
&=\left[\left(\bar{l} \otimes 1+1 \otimes \bar{l}^{\prime}\right),\left(\bar{s} \otimes 1+1 \otimes \bar{s}^{\prime}\right)\right] \\
&=\left(\bar{l} \otimes 1+1 \otimes \bar{l}^{\prime}\right)\left(\bar{s} \otimes 1+1 \otimes \overline{s^{\prime}}\right) \\
&-\sum\left(R^{(2)} \cdot\left(\bar{s} \otimes 1+1 \otimes \overline{s^{\prime}}\right)\right)\left(R^{(1)} \cdot\left(\bar{l} \otimes 1+1 \otimes \bar{l}^{\prime}\right)\right) \\
&=\left(\bar{l} \otimes 1+1 \otimes \bar{l}^{\prime}\right)\left(\bar{s} \otimes 1+1 \otimes \bar{s}^{\prime}\right) \\
&-\sum\left(R^{(2)} \cdot \bar{s} \otimes 1+1 \otimes R^{(2)} \cdot \overline{s^{\prime}}\right)\left(R^{(1)} \cdot \bar{l} \otimes 1+1 \otimes R^{(1)} \cdot \bar{l}^{\prime}\right) \\
&= \sum \bar{l} \bar{s} \otimes 1+\bar{l} \otimes \overline{s^{\prime}}+R^{(2)} \cdot \bar{s} \otimes R^{(1)} \cdot \bar{l}^{\prime}+1 \otimes \bar{l}^{\prime} \bar{s}^{\prime} \\
&-\sum\left(\left(R^{(2)} \cdot \bar{s}\right)\left(R^{(1)} \cdot \bar{l}\right) \otimes 1+R^{(2)} \cdot \bar{s} \otimes R^{(1)} \cdot \bar{l}^{\prime}\right. \\
&\left.\quad+r^{(2)} R^{(1)} \cdot \bar{l} \otimes r^{(1)} R^{(2)} \cdot \overline{s^{\prime}}+1 \otimes\left(R^{(2)} \cdot \overline{s^{\prime}}\right)\left(R^{(1)} \cdot \bar{l}^{\prime}\right)\right) \\
&= {[\bar{l}, \bar{s}] \otimes 1+1 \otimes\left[\bar{l}^{\prime}, \overline{s^{\prime}}\right] \quad[\text { using triangularity of } R] . }
\end{aligned}
$$

Thus $f$ is an $R$-Lie map.

Now by the universal property of $U\left(L_{1} \oplus L_{2}\right)$ there exists an $H_{1}$-module algebra homomorphism $g: U\left(L_{1} \oplus L_{2}\right) \rightarrow U\left(L_{1}\right) \otimes U\left(L_{2}\right)$ as claimed.

Theorem 2.6. Let $\left(H_{1}, R\right)$ be a triangular Hopf algebra, and let $\left(L,[,]_{R}\right)$ be an R-Lie algebra. Let $(U(L), u)$ be the R-universal enveloping algebra of Proposition 2.4. Then $U(L)$ is a Hopf algebra in the category ${ }_{H_{1}}$ Mod with

$$
\begin{aligned}
\Delta(\bar{l}) & =\bar{l} \otimes 1+1 \otimes \bar{l}, & & \\
S(\bar{l}) & =-\bar{l}, & S(\bar{b} \bar{c}) & =\sum\left(R^{(2)} \cdot S(\bar{c})\right)\left(R^{(1)} \cdot S(\bar{b})\right), \\
\varepsilon(\bar{l}) & =0, & \varepsilon(1) & =1,
\end{aligned}
$$

for all $l \in L$ and $\bar{b}, \bar{c} \in U(L)$.

Proof. Analogous to the proof in the standard case. Let $d: L \rightarrow L \oplus L$ be the diagonal map $l \rightarrow(l, l) ; d$ is obviously an $R$-Lie map. Let $f$ be the map described in Proposition 2.5. Then $f \circ d: L \rightarrow(U(L) \otimes U(L))^{-}$is an $R$ Lie homomorphism; hence, there exists an $H_{1}$-module algebra homomorphism $\Delta: U(L) \rightarrow U(L) \otimes U(L)$ satisfying

$$
\Delta(\bar{l})=((f \circ d) l)=\bar{l} \otimes 1+1 \otimes \bar{l}, \quad \text { for all } l \in L .
$$

It is straightforward to check that (id $\otimes \Delta) \circ \Delta=(\Delta \otimes$ id) $\circ \Delta$ and $(\varepsilon \otimes$ id $) \circ \Delta=$ (id $\otimes \varepsilon) \circ \Delta=$ id. Now consider $S$ which is defined on generators.

$$
S(\bar{f})=-\bar{f}, \quad S(1)=1,
$$

and extended to $U(L)$ via:

$$
S(\bar{b} \bar{c})=\sum\left(R^{(2)} \cdot S(\bar{c})\right)\left(R^{(1)} \cdot S(\bar{b})\right), \text { for } \bar{b}, \bar{c} \in U(L) .
$$


It is easy to see that $S$ is a well-defined $H_{1}$-module homomorphism, since if we define $\widetilde{S}$ on the free generators of $T(L)$ by $\widetilde{S}(l)=-l, \widetilde{S}(1)=1$, and set $\widetilde{S}(b c)=\sum\left(R^{(2)} \cdot S(c)\right)\left(R^{(1)} \cdot S(b)\right)$, then $\widetilde{S}$ is an $H_{1}$-module homomorphism which vanishes on $I$. Thus $S$ is well defined.

To show that $S$ is an antipode, we first note that for generators $\bar{l} \in \bar{L}$ one has

$$
\begin{aligned}
(\mu(\mathrm{id} \otimes S) \circ \Delta)(\bar{l}) & =(\mu(S \otimes \mathrm{id}) \circ \Delta)(\bar{l})=0=\varepsilon(\bar{l}), \\
(\mu(\mathrm{id} \otimes S) \circ \Delta)(1) & =(\mu(S \otimes \mathrm{id}) \circ \Delta)(1)=1=\varepsilon(1),
\end{aligned}
$$

which is a straightforward computation, and then we use the following

Claim. If $H$ is a bialgebra in the category ${ }_{H_{1}}$ Mod and if

$$
(\mu(\mathrm{id} \otimes S) \circ \Delta)(a)=\varepsilon(a), \quad(\mu(\mathrm{id} \otimes S) \circ \Delta)(b)=\varepsilon(b),
$$

and $S(a b)=\sum\left(R^{(2)} \cdot S(b)\right)\left(R^{(1)} \cdot S(a)\right)$, then $(\mu(\operatorname{id} \otimes S) \circ \Delta)(a b)=\varepsilon(a b)$.

Proof of Claim.

$$
\begin{aligned}
(\mu(\mathrm{id} & \otimes S) \circ \Delta)(a b) \\
& =\mu(\mathrm{id} \otimes S) \sum\left(a_{(1)}\left(R^{(2)} \cdot b_{(1)}\right) \otimes\left(R^{(1)} \cdot a_{(2)}\right) b_{(2)}\right) \\
& =\mu \sum\left(a_{(1)}\left(R^{(2)} \cdot b_{(1)}\right) \otimes r^{(2)} \cdot S\left(b_{(2)}\right)\left(r^{(1)} R^{(1)} \cdot S\left(a_{(2)}\right)\right)\right) \\
& =\sum a_{(1)}\left(R_{(1)}^{(2)} \cdot b_{(1)}\right)\left(R_{(2)}^{(2)} \cdot S\left(b_{(2)}\right)\right)\left(R^{(1)} \cdot S\left(a_{(2)}\right)\right)(\text { using property }(2) \text { of } R) \\
& =\sum a_{(1)}\left(R^{(2)} \cdot\left(b_{(1)} S\left(b_{(2)}\right)\right)\right)\left(R^{(1)} \cdot S\left(a_{(2)}\right)\right) \\
& =\sum a_{(1)}\left(R^{(2)} \cdot \varepsilon(b)\right)\left(R^{(1)} \cdot S\left(a_{(2)}\right)\right) \quad(\text { by assumption) } \\
& =\varepsilon(a) \varepsilon(b) \quad\left(\text { since } \sum \varepsilon\left(R^{(2)}\right) R^{(1)}=1\right) .
\end{aligned}
$$

Using the claim we deduce that $S$ is an antipode on $U(L)$.

Remark 2.7. If $A$ is an $H$-module algebra and $A^{-}$its derived Lie algebra, then the map $u: A^{-} \rightarrow U\left(A^{-}\right)$is an injection.

Proof. The identity map from $A^{-}$to $A^{-}$is a Lie map; hence by the universality of $U(R)$ there is an algebra map $p: U\left(A^{-}\right) \rightarrow A$ such that $p \circ u=i d$. Thus $u$ is $1-1$.

\section{AN $R$-ANALOGUE OF SCHUR'S DOUBLER CENTRALIZER THEOREM}

Let us first recall Schur's double centralizer theorem in its classical form.

Let $V$ be a finite-dimensional vector space over a field $k$ of characteristic 0 . Let $m$ be a positive integer, and define a representation $\theta$ of the symmetric group $S_{m}$ on $V^{\otimes m}$ by $\sigma \cdot\left(v_{1} \otimes \cdots \otimes v_{m}\right)=v_{\sigma^{-1}(1)} \otimes \cdots \otimes v_{\sigma^{-1}(m)}$. In particular, $(i, i+1) \cdot\left(v_{1} \otimes \cdots \otimes v_{m}\right)=v_{1} \otimes \cdots \otimes v_{i+1} \otimes v_{1} \otimes \cdots \otimes v_{m}$; that is, this representation is essentially defined by the twist map $\tau$. It extends to an action of the Hopf algebra $k S_{m}$ on $V^{\otimes m}$ and hence on $\operatorname{End}_{k} V^{\otimes m}$ (as in (1.1)). On the other hand consider the Hopf algebra $U(\operatorname{gl}(V))$, the universal enveloping algebra of the Lie algebra $\mathrm{gl}(V)$. It acts on $V$ by composition, and since it is a Hopf algebra, it acts on $V^{\otimes m}$ by (1.2). We denote this representation by $\rho^{m}$. It entails using $\Delta^{m-1}(h)$, for all $h \in U(\mathrm{gl}(V))$. 
Theorem 3.1 (Schur's double centralizer theorem in equivalent form). In the above notation we have

$$
\operatorname{End}_{\theta\left(k S_{m}\right)} V^{\otimes m}=\rho^{m}(U(\operatorname{gl}(V))) \text { and } \operatorname{End}_{\rho^{m}(U(\operatorname{gl}(V)))} V^{\otimes m}=\theta\left(k S_{m}\right) .
$$

Berele and Regev [BR] have extended this to super vector spaces, and [Fi] proved their result in a Hopf algebraic setting. In this paper we prove a double centralizer theorem for finite-dimensional $V \in{ }_{H_{1}} \operatorname{Mod}$, where $\left(H_{1}, R\right)$ is a triangular Hopf algebra. The previous results will follow immediately from this theorem. Let us apply the above.

Let $V \in{ }_{H_{1}}$ Mod be a finite-dimensional vector space over $k$. By (1.1) $\operatorname{End}_{k} V \in{ }_{H_{1}} \operatorname{Mod}$. Let $\operatorname{gl}_{R}(V) \equiv\left(\operatorname{End}_{k} V\right)^{-}$, and let $U\left(\mathrm{gl}_{R}(V)\right)$ be the $R$ universal enveloping algebra described in Theorem 2.6.

Remark 3.2. In particular, if $H_{1}=k$, then $U\left(\mathrm{gl}_{R}(V)\right)$ is just $U(\mathrm{gl}(V))$. As for the super case, following Majid's construction [Maj2], let $H_{1}=k \mathbb{Z}_{2}($ char $k \neq 2)$ be the group algebra of the group $\mathbb{Z}_{2}=\{1, g\}$ with the nonstandard triangular structure

$$
R=\frac{1}{2}(1 \otimes 1+1 \otimes g+g \otimes 1-g \otimes g) .
$$

The category of $k \mathbb{Z}_{2}$-modules is precisely the category of super vector spaces, where $g \cdot v=(-1)^{|v|} v$ (where $|v|=$ degree $v$ ).

If $V \in{ }_{k \mathbf{Z}_{2}}$ Mod is finite dimensional, then $U\left(\mathrm{gl}_{R}(V)\right)=U(p l(V))$, where $\operatorname{pl}(V)$ is the Lie superalgebra of $\mathbb{Z}_{2}$-graded endomorphisms of $V$.

Combining Theorem 2.6 and the bosonization theorem we have

Corollary 3.3. Let $\left(H_{1}, R\right)$ and $U\left(\mathrm{gl}_{R}(V)\right)$ be as in Theorem 2.6 with $\mathrm{gl}_{R}(V)=$ $\left(\operatorname{End}_{k} V\right)^{-}, V$ a finite-dimensional vector space over $k$. Then

$$
H=U\left(\mathrm{gl}_{R}(V)\right) \# H_{1}
$$

is an ordinary triangular Hopf algebra with

$$
\begin{gathered}
\Delta(f \# 1)=(f \# 1) \otimes(1 \# 1)+\sum\left(1 \# R^{(2)}\right) \otimes\left(R^{(1)} \cdot f \# 1\right), \quad \Delta(1 \# h)=\Delta_{H_{1}}(h), \\
S(f \# 1)=-\sum y R^{(1)} \cdot f \# S\left(R^{(2)}\right) \quad\left(\text { where } y=\sum S\left(R^{(2)}\right) R^{(1)}\right), \\
S(1 \# h)=1 \# S(h), \quad \varepsilon(f \# 1)=0, \quad \varepsilon(1 \# h)=\varepsilon(h), \quad R_{H}=R_{H_{1}},
\end{gathered}
$$

for all $f \in \mathrm{gl}_{R}(V), h \in H_{1}$.

Since $u: \mathrm{gl}_{R}(V) \rightarrow U\left(\mathrm{gl}_{R}(V)\right)$ is an injection (see Remark 2.7) we write $f$ for $\bar{f}$. Define an obvious representation of $H$ on $V \rho: H \rightarrow \operatorname{End}_{k}(V)$ given by

$$
\rho\left(f_{1} \cdots f_{t} \# 1\right)=f_{1} \circ \cdots \circ f_{t}, \quad \text { for all } f_{i} \in \operatorname{End}_{k} V
$$

and

$$
(\rho(1 \# h))(v)=h \cdot v, \quad \text { for all } h \in H_{1}, v \in V .
$$

Extend $\rho$ to $H$ by $\rho(b \# h)=\rho(b) \circ \rho(h)$, for all $b=f_{1} \cdots f_{t} \in U\left(\mathrm{gl}_{R}(V)\right), h \in$ $H_{1}$. To show that $\rho$ is a representation one has to show that $\rho((1 \# h)(b \# 1))=$ $\rho(h) \circ \rho(b)$. Indeed,

$$
(1 \# h)(b \# 1)=\sum h_{(1)} \cdot b \# h_{(2)} .
$$


Hence

$$
\begin{aligned}
(\rho(1 \# h)(b \# 1))(v) & =\sum\left(\rho\left(h_{(1)} \cdot b\right) \rho\left(h_{(2)}\right)\right)(v) \\
& =\sum\left(h_{(1)} \cdot\left(f_{1} \circ \cdots \circ f_{t}\right)\right)\left(h_{(2)} \cdot v\right) \\
& =\sum h_{(1)} \cdot\left(f_{1} \cdots f_{t}\left(S h_{(2)} h_{(3)} \cdot v\right)\right) \\
& =h \cdot\left(f_{1} \cdots f_{t}(v)\right)=(\rho(h) \circ \rho(b))(v) .
\end{aligned}
$$

Thus $\rho$ is a representation of $U\left(\mathrm{gl}_{R}(V)\right) \# H_{1}$ on $V$. From now on, denote $\rho(g)$ by $\underline{g}$ for all $g \in U\left(\mathrm{gl}_{R}(V)\right) \# H_{1}$; and since $\rho$ is onto we will denote by $\underline{f}$ all elements of $\operatorname{End}_{k} V$. The representation $\rho$ induces a representation $\rho^{m}$ on $V^{\otimes m}$ as in (1.2); i.e.,

$$
\begin{array}{r}
\rho^{m}(g)\left(v_{1} \otimes \cdots \otimes v_{m}\right)=\underline{g_{(1)}}\left(v_{1}\right) \otimes \cdots \otimes \underline{g_{(m)}}\left(v_{m}\right), \\
\text { where } \Delta^{m-1}(g)=\sum g_{(1)} \otimes \cdots \otimes g_{(m)} .
\end{array}
$$

Remark. Some caution is necessary here, for if $x=b \# h \in H$, then one must distinguish between $x$ and $\underline{x} \# 1 ; \Delta(x)$ could very well be different from $\Delta(\underline{x} \# 1)$, and so $\rho^{m}(x) \neq \rho^{m}(\underline{x} \# 1)$. Even in the trivial example if $x=1 \# 1$, then $\underline{x} \# 1=\mathrm{id} \# 1$ and thus

$$
\Delta(\underline{x} \# 1)=(\mathrm{id} \# 1) \otimes(1 \# 1)+(1 \# 1) \otimes(\mathrm{id} \# 1) .
$$

So $\rho^{2}(\underline{x} \# 1)\left(v_{1} \otimes v_{2}\right)=2\left(v_{1} \otimes v_{2}\right)$ while $\Delta(x)=(1 \# 1) \otimes(1 \# 1)$, and so

$$
\rho^{2}(x)\left(v_{1} \otimes v_{2}\right)=v_{1} \otimes v_{2}
$$

(this of course is well known in the representation theory of Lie algebras).

Let us now consider the symmetric group $S_{m}$. There is a representation of $S_{m}$ on $V^{\otimes m}$ induced by $\psi_{V, V}$ (rather than $\tau$ ). Specifically, define $\phi: k S_{m} \rightarrow$ End $V^{\otimes m}$ by

$$
(i, i+1) \cdot\left(v_{1} \otimes \cdots \otimes v_{m}\right)=\sum v_{1} \otimes \cdots \otimes R^{(2)} \cdot v_{i+1} \otimes R^{(1)} \cdot v_{1} \otimes \cdots \otimes v_{m}
$$

extend this multiplicatively to $S_{m}$ and linearly to $k S_{m}$.

The action of the Hopf algebra $k S_{m}$ on $V^{\otimes m}$ now induces an action of $k S_{m}$ on $\operatorname{End}_{k} V^{\otimes m}$ as in (1.1). That is, for all $\underline{f_{j}} \in \operatorname{End}_{k} V$ and $v_{j} \in V$ let

$$
\begin{aligned}
((i & \left., i+1) \cdot\left(\underline{f_{i}} \otimes \cdots \otimes \underline{f_{m}}\right)\right)\left(v_{1} \otimes \cdots \otimes v_{m}\right) \\
& =(i, i+1) \cdot\left(\left(\underline{f_{1}} \otimes \cdots \otimes \underline{f_{m}}\right)\left((i, i+1) \cdot\left(v_{1} \otimes \cdots \otimes v_{m}\right)\right)\right) \\
& =(i, i+1) \cdot\left(\left(\underline{f_{1}} \otimes \cdots \otimes \underline{f_{m}}\right) \sum\left(v_{1} \otimes \cdots \otimes R^{(2)} \cdot v_{i+1} \otimes R^{(1)} \cdot v_{i} \otimes \cdots \otimes v_{m}\right)\right) \\
& =\sum \underline{f_{1}}\left(v_{1}\right) \otimes \cdots \otimes r^{(2)} \cdot\left(\underline{f_{i+1}}\left(R^{(1)} \cdot v_{i}\right)\right) \otimes r^{(1)} \cdot\left(\underline{f_{i}}\left(R^{(2)} \cdot v_{i+1}\right)\right) \otimes \cdots \otimes \underline{f_{m}}\left(v_{m}\right) \\
& =\sum\left(\underline{f_{1}} \otimes \cdots \otimes \underline{r}^{(2)} \underline{f_{i+1}} \underline{R}^{(1)} \otimes \underline{r}^{(1)} \underline{f}_{i} \underline{R}^{(2)} \otimes \cdots \otimes \underline{f_{m}}\right)\left(v_{1} \otimes \cdots \otimes v_{m}\right)
\end{aligned}
$$

(with $r=R$ ). Thus we have shown:

$$
(i, i+1) \cdot\left(\underline{f_{1}} \otimes \cdots \otimes \underline{f_{m}}\right)=\sum \underline{f}_{1} \otimes \cdots \otimes \underline{r}^{(2)} \underline{f}_{i+1} \underline{R}^{(1)} \otimes \underline{r}^{(1)} \underline{f}_{i} \underline{R}^{(2)} \otimes \underline{f}_{i+1} \otimes \cdots \otimes \underline{f_{m}} .
$$

In the following lemma we have repeated occurrences of $R$ which we shall denote by $R_{1}, \ldots, R_{j}$, where $R=R_{i}$, for all $i$. As a matter of convenience we shall write $R_{0}^{(2)}$ for empty word and $R_{0}^{(1)}$ for 1 . 
Lemma 3.7. Let $f \in \mathrm{gl}_{R}(V)$, and let $\Delta=\Delta_{H}$, where $H=U\left(\mathrm{gl}_{R}(V)\right) \# H_{1}$. Then

(1) $\Delta^{m}(f \# 1)=\sum_{j=0}^{m}\left(1 \# R_{1}^{(2)}\right) \otimes \cdots \otimes\left(1 \# R_{j}^{(2)}\right) \otimes\left(R_{j}^{(1)} \cdots R_{1}^{(1)} \cdot f \# 1\right) \otimes 1^{\otimes m-j}$,

(2) $\rho^{m+1}(f \# 1)=(1+(2,1)+\cdots+(m+1, m) \cdots(2,1)) \cdot\left(\underline{f} \otimes \mathrm{id}^{\otimes m}\right)$.

Proof. First we claim that

$$
\begin{aligned}
& \left.(i, i+1) \cdot \underline{f_{1}} \otimes \cdots \otimes \underline{f_{i}} \otimes \mathrm{id} \otimes \cdots \otimes \underline{f_{m}}\right) \\
& =\sum \underline{f_{1}} \otimes \cdots \otimes \underline{f_{i-1}} \otimes R^{(2)} \otimes R^{(1)} \cdot \underline{f_{i}} \otimes \underline{f_{i+1}} \otimes \cdots \otimes \underline{f_{m}} .
\end{aligned}
$$

Well, by (3.5)

$$
\begin{aligned}
(i, i+1) \cdot\left(\underline{f_{1}} \otimes \cdots \otimes \underline{f}_{i} \otimes \mathrm{id} \otimes \cdots \otimes \underline{f_{m}}\right) \\
\quad=\sum \underline{f_{1}} \otimes \cdots \otimes \underline{r}^{(2)} \underline{\mathrm{id}}^{(1)} \otimes \underline{r}^{(1)} \underline{f}_{i} \underline{R}^{(2)} \otimes \underline{f_{i+2}} \otimes \cdots \otimes \underline{f_{m}},
\end{aligned}
$$

so (*) will follow if we show that $\sum \underline{r}^{(2)} \underline{R}^{(1)} \otimes \underline{r}^{(1)} \underline{f} \underline{R}^{(2)}=\sum \underline{R}^{(2)} \otimes \underline{R}^{(1)} \cdot \underline{f}$, for all $\underline{f} \in \operatorname{End}_{k} V$. Now $\sum\left(\underline{R}^{(2)} \otimes R^{(1)} \cdot \underline{f}\right)\left(v_{1} \otimes v_{2}\right)=\sum R^{(2)} \cdot v_{1} \otimes R_{(1)}^{(1)} \cdot\left(\underline{f}\left(S R_{(2)}^{(1)} \cdot v_{2}\right)\right)=$ $\sum R^{(2)} r^{(2)} \cdot v_{1} \otimes R^{(1)} \cdot\left(f\left(S r^{(1)} \cdot v_{2}\right)\right)$ (by property (2) of $\left.R\right)$. But since $(H, R)$ is triangular, $\sum S\left(r^{(1)}\right) \otimes r^{(2)}=R^{-1}=R^{\tau}=\sum r^{(2)} \otimes r^{(1)}$. Substituting this, the above equals

$$
\sum R^{(2)} r^{(1)} \cdot v_{1} \otimes R^{(1)} \cdot\left(\underline{f}\left(r^{(2)} \cdot v_{2}\right)\right)=\sum\left(\underline{R}^{(2)} \underline{r}^{(1)} \otimes \underline{R}^{(1)} \underline{f}^{(2)}\right)\left(v_{1} \otimes v_{2}\right),
$$

as claimed.

Now we prove the lemma by induction on $m$.

For $m=1, \Delta(f \# 1)=(f \# 1) \otimes 1+\sum\left(1 \# R^{(2)}\right) \otimes\left(R^{(1)} \cdot f \# 1\right)$. Hence

$$
\begin{aligned}
\rho^{2}(\underline{f \# 1)} & =\underline{f} \otimes \mathrm{id}+\sum \underline{R}^{(2)} \otimes R^{(1)} \cdot \underline{f} \\
& =1(\underline{f} \otimes \underline{\mathrm{id}})+(2,1) \cdot(\underline{f} \otimes \underline{\mathrm{id}}) \quad(\mathrm{by}(*)) \\
& =(1+(2,1)) \cdot(\underline{f} \otimes \underline{\mathrm{id}})
\end{aligned}
$$

Assume the lemma is true for $\Delta^{m-1}$ and $\rho^{m}$. Then

$$
\begin{aligned}
\Delta^{m} & (f \# 1)=\left(\mathrm{id}^{\otimes m-1} \otimes \Delta\right)\left(\Delta^{m-1}(f)\right) \\
= & \left(\mathrm{id}^{\otimes m-1} \otimes \Delta\right) \sum_{j=0}^{m-1}\left(1 \# R_{1}^{(2)}\right) \otimes \cdots \otimes\left(1 \# R_{j}^{(2)}\right) \otimes\left(R_{j}^{(1)} \cdots R_{1}^{(1)} \cdot f \# 1\right) \otimes 1^{\otimes m-1-j} \\
= & \left(\mathrm{id}^{\otimes m-1} \otimes \Delta\right)\left(\sum_{j=0}^{m-2}\left(1 \# R_{1}^{(2)}\right) \otimes \cdots \otimes\left(1 \# R_{j}^{(2)}\right) \otimes\left(R_{j}^{(1)} \cdots R_{1}^{(1)} \cdot f \# 1\right) \otimes 1^{\otimes m-1-j}\right) \\
& +\left(1 \# R_{1}^{(2)}\right) \otimes \cdots \otimes\left(1 \# R_{m-1}^{(2)}\right) \otimes\left(R_{m-1}^{(1)} \cdots R_{1}^{(1)} \cdot f \# 1\right) \\
= & \sum_{j=0}^{m-2}\left(1 \# R_{1}^{(2)}\right) \otimes \cdots \otimes\left(1 \# R_{j}^{(2)}\right) \otimes\left(R_{j}^{(1)} \cdots R_{1}^{(1)} \cdot f \# 1\right) \otimes 1^{\otimes m-j-1} \\
& +\left(1 \# R_{1}^{(2)}\right) \otimes \cdots \otimes\left(1 \# R_{m-1}^{(2)}\right) \otimes \Delta\left(R_{m-1}^{(1)} \cdots R_{1}^{(1)} \cdot f \# 1\right) .
\end{aligned}
$$

But

$$
\begin{aligned}
\Delta\left(R_{m-1}^{(1)} \cdots R_{1}^{(1)} \cdot f \# 1\right)= & \left(\left(R_{m-1}^{(1)} \cdots R_{1}^{(1)} \cdot f \# 1\right) \otimes 1\right) \\
& +\left(1 \# R_{m}^{(2)}\right) \otimes\left(R_{m}^{(1)} R_{m-1}^{(1)} \cdots R_{1}^{(1)} \cdot f \# 1\right) .
\end{aligned}
$$


Hence, the whole expression equals

$$
\sum_{j=0}^{m}\left(1 \# R_{1}^{(2)}\right) \otimes \cdots \otimes\left(1 \# R_{j}^{(2)}\right) \otimes\left(R_{j}^{(1)} \cdots R_{1}^{(1)} \cdot f \# 1\right) \otimes 1^{\otimes m-j} .
$$

We have proved (1). Now, for (2) we show by induction that

$$
(* *)(m+1, m) \cdots(2,1) \cdot\left(\underline{f} \otimes \underline{\mathrm{id}}^{\otimes m}\right)=\sum \underline{R}_{1}^{(2)} \otimes \cdots \otimes \underline{R}_{m}^{(2)} \otimes R_{m}^{(1)} \cdots R_{1}^{(1)} \cdot \underline{f} .
$$

This is true for $m=1$; assume for $m$. Then

$$
\begin{aligned}
& (m+1, m) \cdots(2,1)\left(\underline{f} \otimes \underline{\mathrm{id}}^{\otimes m}\right) \\
& =(m+1, m) \cdot\left((m, m-1) \cdots(2,1) \cdot\left(\underline{f} \otimes \underline{\mathrm{id}}^{\otimes m-1} \otimes \underline{\mathrm{id}}\right)\right) \\
& =(m+1, m) \cdot \sum\left(\underline{R}_{1}^{(2)} \otimes \cdots \otimes \underline{R}_{m-1}^{(2)} \otimes R_{m-1}^{(1)} \cdots R_{1}^{(1)} \cdot \underline{f} \otimes \underline{\mathrm{id}}\right) \\
& \text { (by the induction hypothesis) } \\
& =\sum \underline{R}_{1}^{(2)} \otimes \cdots \otimes \underline{R}_{m-1}^{(2)} \otimes \underline{R}_{m}^{(2)} \otimes R_{m}^{(1)} \cdots R_{1}^{(1)} \cdot \underline{f} \quad(\text { by }(*)), \\
& {[1+(2,1)+\cdots+(m, m-1) \cdots(2,1)+(m+1, m) \cdots(2,1)] \cdot\left(\underline{f} \otimes \underline{\mathrm{id}}^{\otimes m}\right)} \\
& \left.=\rho^{m}(f \# 1) \otimes \mathrm{id}+((m+1, m) \cdots(2,1)) \cdot\left(\underline{f} \otimes \underline{\mathrm{id}}^{\otimes m}\right) \quad \text { (by induction }\right) \\
& =\rho^{m}(f \# 1) \otimes \underline{\mathrm{id}}+\underline{R}_{1}^{(2)} \otimes \cdots \otimes \underline{R}_{m}^{(2)} \otimes R_{m}^{(1)} \cdots R_{1}^{(1)} \cdot \underline{f} \quad(\text { by }(* *)) \\
& =\sum_{j=0}^{m-1} \underline{R}_{1}^{(2)} \otimes \cdots \otimes \underline{R}_{j}^{(2)} \otimes R_{j}^{(1)} \cdots R_{1}^{(1)} \cdot \underline{f} \otimes \underline{\mathrm{id}}^{\otimes m-j} \\
& +\underline{R}_{1}^{(2)} \otimes \cdots \otimes \underline{R}_{m}^{(2)} \otimes R_{m}^{(1)} \cdots R_{1}^{(1)} \cdot \underline{f}=\rho^{m+1}(f \# 1) \quad(\text { by }(1)) .
\end{aligned}
$$

We are ready to prove the $R$-centralizer theorem; let us recall some facts. If $A$ is a $k S_{m}$ module algebra and $t \in \sum_{\sigma \in S_{m}} \sigma$, then $t \cdot A \subset A^{k S_{m}}$. We say $A$ has an element of trace 1 if there exists $a \in A$ with $t \cdot a=1$, and then $A^{k S_{m}}=t \cdot A$. In particular, if char $k=0$ and $A=\operatorname{End}_{k} V$, then $t(1 / m !)=1$.

Theorem 3.8. Let $\left(H_{1}, R\right)$ be a triangular Hopf algebra, $V \in H_{1}$ Mod a finitedimensional vector space over a field $k$ of characteristic 0 , and $m \in \mathbb{N}$. Let $\rho^{m}: H \rightarrow \operatorname{End}_{k} V^{\otimes m}$ and $\phi: k S_{m} \rightarrow \operatorname{End}_{k} V^{\otimes m}$ be the representations described in (3.4) and (3.5). Then

(1) $\operatorname{End}_{\phi\left(k S_{m}\right)} V^{\otimes m}=\rho^{m}(H)$,

(2) $\operatorname{End}_{\rho^{m}(H)} V^{\otimes m}=\phi\left(k S_{m}\right)$.

Proof. The proof closely follows the proof in [Fi].

(0) Let $k S_{m}$ act on End $_{k} V^{\otimes m}$ as in (3.6).

(1) Since there exists a trace 1 element in $\operatorname{End}_{k} V^{\otimes m}$, we have $\left(\operatorname{End}_{k} V^{\otimes m}\right)^{k S_{m}}$ $=t \cdot\left(\operatorname{End}_{k} V^{\otimes m}\right)$. However, $\left(\operatorname{End}_{k} V^{\otimes m}\right)^{k S_{m}}=\operatorname{End}_{\phi\left(k S_{m}\right)} V^{\otimes m}$. Thus to show (1) we need to show that $t \cdot\left(\operatorname{End}_{k} V^{\otimes m}\right)=\rho^{m}(H)$.

$$
\text { Now, } \rho^{m}(H) \subset \operatorname{End}_{\phi\left(k S_{m}\right)} V^{\otimes m} \text { by [Fi, 2.1]. }
$$

So we show the reverse inclusion by induction on $m$. For $m=1, \operatorname{End}_{\phi\left(k S_{1}\right)} V$ $=\operatorname{End}_{k} V=\rho(H)$. Assume the inclusion for $m-1$. Let $a=1+(2,1)+\cdots+$ 
$((m, m-1) \cdots(2,1)) \in k S_{m}$ and $t^{\prime}=\sum_{\sigma \in S_{\{2, \ldots, m\}}} \sigma$. Then $t=a t^{\prime}$, and thus, for all $\underline{f_{1}} \otimes \cdots \otimes \underline{f_{m}} \in \operatorname{End}_{k} V^{\otimes m}, t \cdot\left(\underline{f_{1}} \otimes \cdots \otimes \underline{f_{m}}\right)=a t^{\prime} \cdot\left(\underline{f_{1}} \otimes \cdots \otimes \underline{f_{m}}\right)=a \cdot$ $\left(\underline{f_{1}} \otimes t^{\prime} \cdot\left(\underline{f_{2}} \otimes \cdots \otimes \underline{f_{m}}\right)\right)$. But $t^{\prime} \cdot\left(\underline{f_{2}} \otimes \cdots \otimes f_{m}\right) \in \rho^{m-1}(H)$ by the induction hypothesis. Hence there exists a $g=\sum b_{i} \# \bar{h}_{i} \in H$ with $t^{\prime} \cdot\left(\underline{f_{2}} \otimes \cdots \otimes \underline{f_{m}}\right)=$ $\rho^{m-1}(g)$. If $\Delta^{m-2}(g)=\sum g_{(1)} \otimes \cdots \otimes g_{(m-1)}$, then the above equals

$$
\begin{aligned}
a \cdot \sum & \left(\underline{f_{1}} \otimes \underline{g}_{(1)} \otimes \cdots \otimes \underline{g_{(m-1)}}\right) \\
& =a \cdot \sum\left(\underline{f_{1} S g} \underline{g}_{(1)} \underline{g}_{(2)} \otimes \underline{g}_{(3)} \otimes \cdots \otimes \underline{g_{(m+1)}}\right) \\
& =a \cdot\left(\sum\left(\underline{f_{1} S g_{(1)}} \otimes \underline{\mathrm{id}}^{\otimes m-1}\right)\left(\underline{g_{(2)}} \otimes \cdots \otimes \underline{g_{(m+1)}}\right)\right) \\
& =a \cdot\left(\sum\left(\left(\underline{f_{1} S g_{(1)}}\right) \otimes \mathrm{id}^{m-1}\right) \rho^{m}\left(g_{(2)}\right)\right) .
\end{aligned}
$$

But by Theorem $3.8 \rho^{m}\left(g_{(2)}\right)$ is fixed by $k S_{m}$; hence the above equals

$$
a \cdot\left(\sum \underline{f_{1} S g_{(1)}} \otimes \underline{\mathrm{id}}^{m-1}\right) \rho^{m}\left(g_{(2)}\right)=\rho^{m} \sum\left(\underline{f_{1} S g_{(1)}} \# 1\right) \rho^{m}\left(g_{(2)}\right)
$$

(by Lemma 3.7) and this is in $\rho(H)$, as claimed.

(2) follows as in [Fi].

Remark 3.10. It is now obvious that both the classical and the "super" double centralizer theorems are special cases of Theorem 3.8 with $H_{1}=k, R=1 \otimes 1$, and $\left.\left(H_{1}, R\right)=k \mathbb{Z}_{2}, \frac{1}{2}(1 \otimes 1+1 \otimes g+g \otimes 1-g \otimes g)\right)$, respectively.

\section{REFERENCES}

[BR] A. Berele and A. Regev, Hook Young diagrams with applications to combinatorics and to representations of Lie superalgebras, Adv. Math. 64 (1987), 118-175.

[CW] M. Cohen and S. Westreich, From sypersymmetry to quantum commutativity, J. Algebra (to appear).

[D] V. Drinfeld, Quantum groups, Proc. Internat. Congr. Math. (Berkeley), Amer. Math. Soc., Providence, RI, 1986, pp. 798-819.

[Fi] D. Fischman, Schur's double centralizer theorem: a Hopf algebra approach, J. Algebra 157 (1993), 331-340.

[Maj1] S. Majid, Quasitriangular Hopf algebras and Yang-Baxter equations, Internat. J. Modern Phys. A 5 (1990), 1-91.

[Maj2] _ Cross products by Braided groups and Bosonization, preprint.

[Man] Y. Manin, Quantum groups and non-commutative geometry, Univ. of Montreal lectures, 1988.

[S] M.E. Sweedler, Hopf algebras, Benjamin, New York, 1969.

(M. Cohen and S. Westreich) Department of Mathematics, Ben Gurion University, BeER SHEVA, ISRAEL

E-mail address, M. Cohen: mia@black.bgu.ac.il

(D. Fischman) Department of Mathematics, University of Southern California, Los ANGeles, California 90089

E-mail address: fischman@math.usc.edu 